\title{
Inovações tecnológicas na abordagem de sífilis adquirida na adolescência para estudantes de uma escola estadual do Pará: um relato de experiência
}

Technological innovations in adolescent acquired syphilis approach for students from a state school in Pará: an experience report

Innovaciones tecnológicas en el enfoque de la sífilis adquirida en adolescentes para estudiantes de una escuela estatal en Pará: un informe de experiencia

Adrielly Cristiny Mendonça Fonseca ${ }^{1 *}$, Alana Beatrice Gonçalves Batista ${ }^{1}$, Alcideli Pinheiro dos Santos ${ }^{1}$, Esteliane da Conceição Leão ${ }^{1}$, Geice Kelly Costa Soares Garcia ${ }^{1}$, Hugo de Paulo Garcia da Costa ${ }^{1}$, Pedrinha do Socorro Castro Prazeres ${ }^{1}$, Ianny Ferreira Raiol ${ }^{1}$, Shirley de Aviz Miranda ${ }^{1}$, Maria Rute de Souza Araújo'.

\section{RESUMO}

Objetivo: Relatar uma ação educativa por meio de tecnologias educacionais sobre sífilis adquirida para estudantes de uma escola estadual do Pará. Relato de Experiência: A construção da atividade foi baseada em três momentos. No primeiro momento foi elaborado e distribuição de um folder educativo para os alunos, no segundo momento foi aplicado uma tecnologia adaptada para a realidade dos estudantes elaborada pelos autores, denominado jogo da memória e, no terceiro momento foi utilizado como atividade complementar a dinâmica dos balões onde estimulou-se uma competição de conhecimentos. Participaram da ação educativa 58 alunos, sendo 36 homens e 22 mulheres, na faixa etária prevalente de 15 a 17 anos. Percebeu-se que a maioria dos alunos desconheciam a doença como sexualmente transmissível assim como sua prevenção e a existência do teste rápido como forma de diagnóstico, mas já tinham ouvido falar e sabiam que a sífilis tem cura. Considerações finais: Vale ressaltar a importância de ações que visem à prevenção da doença através de tecnologias inovadoras. Sendo de suma importância para que o processo de ensino-aprendizagem se consolide.

Palavras-chave: Invenções, Sífilis, Adolescente, Educação em saúde.

\begin{abstract}
Objective: Report an educational action through educational technologies about syphilis acquired for students of a state school in Pará. Experience Report: The construction of the activity was based on three moments. At the first moment it was elaborated and distribution of an educational folder for the students, in the second moment it was applied a technology adapted to the reality of the students elaborated by the authors, called memory game and, in the third moment it was used as a complementary activity the dynamics of the balloons. where a competition of knowledge was stimulated. 58 students participated in the educational action, 36 men and 22 women, in the prevalent age group of 15 to 17 years. Most students were unaware of the disease as sexually transmitted, as well as its prevention and the existence of rapid testing as a diagnosis but had heard and knew that syphilis has a cure. Considerations: It is worth mentioning the importance of projects/actions aimed at preventing the disease through innovative technologies. Being extremely important for the teachinglearning process to consolidate.
\end{abstract}

Keywords: Inventions, Syphilis, Adolescent, Health education.

${ }^{1}$ Centro Universitário Metropolitano da Amazônia (UNIFAMAZ), Belém-Pará. *E-mail: adriellycmf@gmail.com SUBMETIDO EM: 10/2019 


\section{RESUMEN}

Objetivo: Informar una acción educativa a través de tecnologías educativas sobre la sífilis adquirida para estudiantes de una escuela estatal en Pará. Informe de experiencia: La construcción de la actividad se basó en tres momentos. En el primer momento se elaboró y distribuyó una carpeta educativa para los alumnos, en el segundo momento se aplicó una tecnología adaptada a la realidad de los alumnos elaborada por los autores, llamada juego de memoria y, en el tercer momento se utilizó como actividad complementaria la dinámica de los globos. donde se estimuló una competencia de conocimiento. 58 estudiantes participaron en la acción educativa, 36 hombres y 22 mujeres, en el grupo de edad predominante de 15 a 17 años. La mayoría de los estudiantes no sabían que la enfermedad se transmitía sexualmente, así como su prevención y la existencia de pruebas rápidas como diagnóstico, pero habían escuchado y sabían que la sífilis tiene una cura. Considerações finais: Vale la pena mencionar la importancia de los proyectos / acciones dirigidos a prevenir la enfermedad a través de tecnologías innovadoras. Ser extremadamente importante para la consolidación del proceso de enseñanza-aprendizaje.

Palabras clave: Invenciones, Sífilis, Adolescente, Educación en salud.

\section{INTRODUÇÃO}

As infecções sexualmente transmissíveis (IST) são doenças infectocontagiosas transmitidas por contato sexual que geralmente se manifestam por meio de lesões cutâneas. Estão entre as mais comuns patologias da saúde pública em todo o mundo, devido à gravidade e prevalência. Estudos registram que mais de 1 milhão de pessoas obtêm uma IST diariamente e estima-se que, a cada ano 500 milhões de pessoas adquirem uma das IST curáveis. Dentre estas infecções destaca-se a sífilis, que possui um preocupante aspecto epidemiológico (ALVES CC, et al., 2019).

A sífilis é uma doença antiga que, recentemente, voltou a preocupar a saúde pública não só no Brasil como em vários países do mundo. Dessa maneira, ela se caracteriza como uma IST curável e exclusiva do ser humano, causada por uma bactéria, o Treponema Pallidum, que passa de pessoa a pessoa pelo contato direto com lesões chamadas de úlceras, as quais ocorrem nos genitais. Sua transmissão ocorre de forma principal com o contato sexual ou vertical de mãe para feto, resultando nas suas formas adquiridas e congênitas respectivamente (KALININ Y, et al., 2017).

Com o crescente número de casos de sífilis que ocorre de forma global notou-se que a doença acomete 12 milhões de pessoas por ano. No Brasil, nos anos de 2010 e 2017, foram notificados no Sistema de Informação de Agravos de Notificação (SINAN) 479.730 casos de sífilis adquirida, evidenciando, um aumento de $31,8 \%$ (de 44,1 para 58,1 casos por 100 mil habitantes). Haja vista que, as taxas de detecção de sífilis adquirida na faixa etária de 13 a 19 anos de idade vem apresentando tendência de aumento desde 2010, no qual o incremento no percentual foi de 39,9\% (SINAN, 2019).

A maioria das pessoas com sífilis tende a não ter conhecimento da infecção, podendo transmiti-la aos seus parceiros logo isso ocorre devido à ausência ou a falta de sintomas da doença, que são apresentados de variáveis formas ao longo de seus estágios e além de tudo tendo seu período de latência (BRASIL, 2018).

Sífilis é uma doença sistêmica de evolução crônica, cujo curso de evolução é variável, dependendo do tempo de contaminação e estágio da doença. A patologia apresenta-se clinicamente subdividida em primária, secundária, latente - recente e tardia - e terciária, os quais apresentam suas peculiaridades clínicas, dependentes do avanço da doença, mas que na maioria dos casos, manifesta-se por lesão única e indolor no genital ou na região que o engloba cuja evolui pra uma afecção local como pápulas e placas mucosas ou sistêmica como febre, mal-estar, cefaléia e ou alterações neurológicas, cardiovasculares e ósseas (CARVALHO GP, 2015). As relações sexuais na adolescência têm iniciado cada vez mais cedo, motivadas pela curiosidade e reforçadas pela necessidade de afirmar sua autonomia. Dessa maneira, as práticas sexuais, dessa população, iniciam sem conhecimento adequado e orientações necessárias a respeito do ato sexual de modo seguro, o que os tornam um alvo fácil ao acometimento de IST (SANTOS DS, et al., 2017).

REAS/EJCH | Vol.Sup.n.41 | e2234 | DOI: https://doi.org/10.25248/reas.e2234.2020 Página 2 de 6 
A população dos adolescentes apresenta baixa adesão ao preservativo e a atividade sexual geralmente não é programada. Estudos revelam que apenas um terço deles usam preservativo durante as relações sexuais. Isso se confirma a partir dos dados da Organização Mundial da Saúde (OMS), a qual grande maioria dos adolescentes inicia sua vida sexual entre 12 e 17 anos (SANTOS DS, et al., 2017).

A estratégia básica de prevenção da transmissão da sífilis, pelos profissionais da saúde, é a informação de forma direcionada a orientar o indivíduo à percepção de fatores de risco, ter um diálogo franco sobre as mudanças no comportamento sexual e adoção do preservativo (LEÃO CC e ABREU RF, 2019). É necessário construir um conhecimento crítico dos adolescentes em relação a sua saúde, favorecer a eles o empoderamento de cuidar de si próprio, os responsabilizando de manter sua saúde e se prevenir contra as infecções sexualmente transmissíveis e de uma gravidez indesejada (SANTOS SB, et al., 2019).

Nesse contexto, o ambiente escolar é essencial para orientação de uma vida sexual adequada. O mesmo caracteriza-se como um local de compromisso e responsabilidade social, aberta aos vários tipos de diálogos e discussões, como, por exemplo, a sexualidade, pois muitos jovens desconhecem seu corpo, por timidez ou vergonha, os mesmos se tornam mais vulneráveis aos riscos inerentes numa relação sexual. Por tanto, o processo educativo torna-se fundamental ao alicerce hábitos e costumes de um grupo ou de um indivíduo, pois assim métodos educativos serão eficazes (LEÃO CC e ABREU RF, 2019).

As tecnologias educacionais tem finalidade de auxiliar no conhecimento e influenciar nas práticas educativas da comunidade. Compreende-se as tecnologias educativas como metodologia inovadora e ferramenta fundamental que possibilita a construção do conhecimento, reflexão e criticidade dos sujeitos, enquanto instrumento emancipador sobre sua vida e saúde, colocando-os como agente ativo e estimulandoos a buscar respostas para problemas reais e complexos. Dessa maneira, a partir desse mecanismo, constróise técnicas e instrumentos integrados ao desenvolvimento dos processos de ensino-aprendizagem capazes de aperfeiçoar uma realidade e alcançar os benefícios que somente o processo educacional pode proporcionar, especialmente em populações vulneráveis (SILVEIRA RSO, et al., 2016). Assim, tem-se que a educação em saúde é uma prática de grande importância para a enfermagem. Como um educador em saúde, o enfermeiro deve sensibilizar o autocuidado e a autonomia nas relações pessoais. As ações que envolvem a prevenção, a promoção e a educação em saúde, quando realizadas com dedicação podem transformar a realidade dos adolescentes (NOBRE CS, et al., 2018). Dessa forma, o estudo propõe descrever uma ação educativa sobre tecnologias educacionais a respeito da Sífilis adquirida para estudante de uma escola estadual do Pará.

\section{RELATO DE EXPERIÊNCIA}

Trata-se de uma ação educativa tipo relato de experiência, sobre inovações tecnológicas, na abordagem de sífilis adquirida na adolescência para estudantes de uma escola em Belém do Pará. A ação educativa foi desenvolvida para alunos de uma escola técnica estadual do Pará. Ocorreu no horário 10:00 às 12:00 horas do dia 17 de outubro de 2019. O público alvo foram 58 estudantes, sendo 36 homens e 22 mulheres, na faixa etária prevalente de 15 a 17 anos.

A ação educativa ocorreu em três momentos: No primeiro momento foi elaborado um folder educativo e distribuído para os alunos, para que pudessem acompanhar a apresentação de uma abordagem geral sobre sífilis. Nesse contexto, o conteúdo do folder abordava sobre o que são IST's, evidenciando a sífilis diante de suas características sintomatológicas clínicas, tipos, estágios, transmissão, diagnóstico, tratamento e prevenção da doença. Além disso, nele continham diversas imagens que contextualizavam com as informações presentes, desse modo, possibilitando a identificação ou suspeita dos sinais e sintomas deste tipo de infecção como forma de conscientização da população sobre os riscos da sífilis. No segundo momento foi aplicado uma tecnologia adaptada para a realidade dos estudantes elaborada pelos autores, denominado jogo da memória, um jogo didático montado em 14 pares que apresentavam fotos e conceitos sobre o tema abordado, para que os estudantes pudessem reconhecer as figuras e relacionar com os respectivos conceitos. O objetivo do jogo foi promover o reconhecimento através do recurso lúdico, onde o ambiente se torna mais atraente, além de servir de motivação e estimulo para o aprendizado. 
No terceiro momento foi utilizado como atividade complementar a dinâmica dos balões onde estimulou-se uma competição de conhecimentos. Ocorreu da seguinte forma: pediu-se para que os estudantes presentes se dividissem em dois grupos, em seguida foram distribuídos balões verde e vermelho para cada estudante, onde verde significa verdadeiro e vermelho falso. Foram escolhidos representantes de cada grupo para que estourassem os balões, que continham perguntas referentes ao assunto abordado. A equipe que respondesse corretamente sobre a informação que continha no balão pontuava. Ao final da dinâmica a equipe que obteve maior pontuação consagrou-se vencedora da competição dos conhecimentos e ganhou brinde. No final de cada dinâmica, foi aberto um momento para discussão de perguntas feitas pelos participantes e conforme os questionamentos apareciam eram solucionados pelos acadêmicos. Durante todos os momentos os participantes fizeram diversas perguntas sobre as IST's, tais como: "Essas doenças podem ser transmitidas por mosquito?"; " Posso pegar uma IST pelo assento sanitário?"; "Uma pessoa pode pegar IST pelo sexo oral?". Diante disso, foi perceptível que os participantes apresentavam pouco conhecimento a respeito da transmissibilidade das infecções.

As principais e mais frequentes dúvidas sobre sífilis estavam voltadas para sua transmissão, diagnóstico e prevenção. Nesse sentido, foram suscitados debates sobre 0 ato sexual e o compartilhamento de objetos pessoais, como roupas íntimas, e outras formas de transmissibilidade, assim como, a conscientização e a facilidade presentes nos métodos sorológicos para o diagnóstico precoce da doença e, por fim, as medidas profiláticas tanto de cuidado pessoal como de alerta ao parceiro em caso de relação sexual ou em outras formas de contágio. Percebeu-se que a maioria dos alunos desconheciam a sífilis como infecção sexualmente transmissível, assim como, sua prevenção e a existência de teste rápido como forma de diagnóstico. Apesar disso, os participantes já tinham ouvido falar da doença e sabiam que esta possui cura. Dessa forma, a atividade permitiu a diminuição da vulnerabilidade desses indivíduos diante da sífilis e a todos os seus agravos.

Desse modo, novas formas de abordar o assunto foram apresentadas e permitiram a expansão do conhecimento, além da possibilidade de busca de novos conhecimentos diante de curiosidades que possam surgir posteriormente. A exemplo disso, o docente, que estava presente durante todo o processo, informou da possibilidade de desenvolvimento de novas atividades a partir dessa em questão, demonstrando a grande viabilidade pedagógica da ação educativa. No final da ação educativa baseada nas dinâmicas utilizadas, percebeu-se que os estudantes adquiriram conhecimentos sobre a sífilis, podendo ser multiplicadores de informações para seus pares, reforçando desta forma a importância da educação em saúde por meio de tecnologias educacionais para os adolescentes.

\section{DISCUSSÃO}

O estudo revelou um conhecimento superficial dos alunos em relação a sífilis como infecção sexualmente transmissível. Segundo Silva GA e Martins MCT (1995) a sexualidade apesar de ser um processo comum da vida, precisa de uma atenção especial e o período da adolescência e fundamental nas descobertas relacionadas ao processo, por isso, esse momento da vida deve receber informações de qualidade sobre 0 assunto. De acordo com Dantas LA, et al. (2017) percebeu-se o aumento nos índices epidemiológicos a respeito da sífilis e outras IST's que atinge grandes partes dos adolescentes, torna-se de extrema importância discutir sobre este assunto.

Estudo realizado por Santos BS, et al. (2019), mostrou que apesar de alguns adolescentes saberem algumas informações sobre as doenças sexualmente transmissíveis, percebeu-se que eram na maioria das vezes informações curtas, equivocadas e que tinham ouvido falar, mas sem saber exatamente suas especificações. O uso de ações educativas é uma forte ferramenta que auxilia na promoção e prevenção de doenças e agravos a saúde. Segundo Teixeira E e Oliveira DC (2014) desenvolver atividades educativas de educação em saúde voltadas para o público adolescente nas escolas, é de extrema importância, visto que essa prática traz informações concretas, auxilia em melhores hábitos de saúde e em práticas preventivas.

Outrossim, Áfio ACE, et al. (2014) ressalta que as tecnologias educativas são instrumentos facilitadores do processo ensino-aprendizagem utilizados como meio de transferência de conhecimento, propiciando ao 
indivíduo a participação em um momento de troca de experiências conducente ao aprimoramento de habilidades. A tecnologia educacional tem o intuito de educar, estimular um pensamento crítico-reflexivo, buscar e/ou amenizar os problemas, visto que, os materiais de ensino dinamizam as atividades de Educação em Saúde.

A importância do uso das tecnologias educativas para a prevenção as doenças, na adolescência, gera conhecimentos necessários para esse público e transforma a realidade da saúde, pois Segundo Bezerra IMP e Sorpreso ICE (2016) educar vai além do saber individual, esta ação gera mudança em conjunto, ajudando na compreensão do indivíduo a respeito de doenças e prevenção das mesmas. Assim, percebe-se a necessidade de desenvolver métodos inovadores como estratégia capaz de promover ações de saúde.

\section{CONSIDERAÇÕES FINAIS}

Observou-se que é necessário alertar mais os adolescentes sobre os cuidados que os mesmos devem tomar em relação a saúde reprodutiva na adolescência. De maneira que a informação é imprescindível para a melhora do conhecimento sendo a ação educativa a ferramenta mais indicada para a promoção da saúde e prevenção de doenças. Salienta-se a necessidade de estudos equivalentes a este para a melhor percepção a respeito da sífilis sendo evidente o interesse de projetos de ação similares que visem à prevenção, estimulando a adesão de medidas simples de controle da doença. Dessa maneira, o uso de tecnologias educativas é essencial, pois têm finalidade de contribuir com atividades de ensino-aprendizagem e mediar práticas educativas em comunidade com tipos específicos de usuários. Além do mais, o uso de tecnologia é essencial como forma de facilitar o entendimento, proporcionar o aprimoramento de habilidades e alcançar os benefícios que somente o processo educacional pode proporcionar. A tecnologia educacional tem o intuito de educar e estimular um pensamento crítico-reflexivo para que as pessoas adquirem autonomia e melhora da sua qualidade de vida.

\section{REFERÊNCIAS}

1. ÁFIO ACE, et al. Analysis of the concept of nursing educational technology applied to the patient. Rev Rene - Revista da Rede de Enfermagem do Nordeste. Universidade Federal do Ceará. Fortaleza, 2014; 15 (1): 58-165.

2. ALVES CC, et al. IST'S na adolescência. In: V Mostra Interdisciplinar do Curso de Enfermagem. Centro Universitário Católica de Quixadá. Quixadá: Ceará. 2019.

3. BEZERRA IMP, SORPRESO ICE. Concepts and movements in health promotion to guide educational 28 practices. J Hum Growth Dev. São Paulo, 2016; 26(1): 11-20.

4. BRASIL, Ministério da Saúde. Boletim epidemiológico Sífilis 2017. Secretaria de Vigilância em Saúde. Brasília - DF: 2017.

5. BRASIL, Ministério da Saúde. Secretaria de Vigilância em Saúde. Departamento de Vigilância, Prevenção e Controle das Infecções Sexualmente Transmissíveis, do HIV/Aids e das Hepatites Virais. Brasília - DF: 2018.

6. CARVALHO GP. Sífilis: A importância investigatória e sua cadeia de transmissão. Dissertação (Mestrado) - Curso de Medicina, Universidade Católica de Pelotas, Pelotas: Rio Grande do Sul, 2015.

7. DANTAS LA, et al. Perfil epidemiológico da Sífilis Adquirida diagnostica e notificada no hospital universitário materno infantil. Enfermería Global, revista eletrônica de enfermagem. Rio grande do Norte, 2017; 46: 227-236.

8. KALININ Y, et al. Sífilis: aspectos clínicos, transmissão, manifestações orais, diagnóstico e tratamento. Revisão bibliográfica. São Paulo, 2015; 23(45-46): 65-76.

9. LEÃO CC, ABREU RF. Assistência de enfermagem preventiva para a sífilis na adolescência. Trabalho de Conclusão de Curso (TCC) - Graduação em Enfermagem, Centro Universitário de Goiás Uni- Anhanguera, Goiânia, 2019.

10. MOROSKOSKI M, et al. Perfil de gestantes adolescentes diagnosticadas com sífilis em Curitiba-PR. R. Saúde Públ. 2018; 1(1):47-58.

11. NOBRE CS, et al. Sistema de saúde no controle da sífilis na perspectiva das enfermeiras [Health system in syphilis control, from the nurses' perspective] [Sistema de salud en el control de la sífilis en la perspectiva de las enfermeras]. Revista Enfermagem Uerj. Universidade do Estado do Rio de Janeiro, 2018; 26: 1-6.

12. SANTOS DS, et al. Sexualidade na adolescência: contaminação de IST's. In: International Nursing Congress, Lisbon: Portugal. 2017; 4: 9 - 12.

13. SANTOS SB, et al. Sífilis adquirida: construção e validação de tecnologia educativa para adolescentes. J Hum Growth Dev. 2019; 29(1): 65-74.

14. Secretaria de Vigilância à Saúde. Sistema de Informação de Agravos de Notificação - Sinan. Normas e Rotinas. Brasília: Ministério da Saúde, 2019. 
15. SILVA GA, MARTINS MCT. Sexualidade na contramão: roteiro de orientação temática para pais, educadores e lideranças comunitárias. São Paulo: Paulus, 1995; 18: 51-69.

16. SILVEIRA RSO, et al. Construção de Tecnologia Educativa para Incentivar Puérperas ao Aleitamento Materno. Ceará, 2016; 1: 1-3.

17. TEXEIRA E, OLIVEIRA DC. Representações sociais de educação em saúde em tempos de AIDS. Rev Bras Enferm. 2014; 67(5): 810-17. 